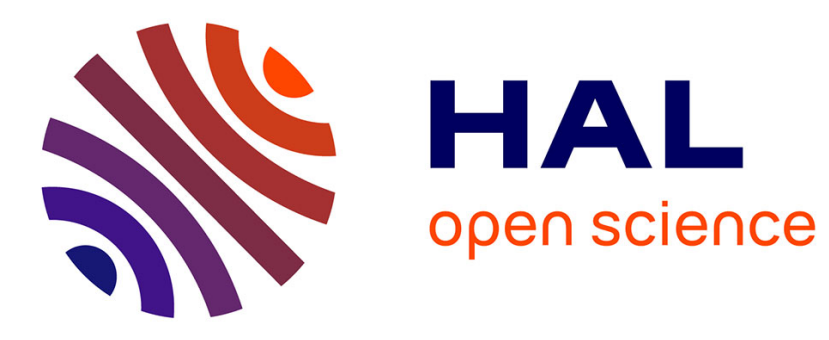

\title{
Doxorubicin-induced cardiac dysfunction is attenuated by cyclosporine treatment in mice through improvements in mitochondrial bioenergetics
}

Xavier Marechal, David Montaigne, Camille Marciniak, Philippe Marchetti, Sidi Mohamed Hassoun, Jean Claude Beauvillain, Steve Lancel, Remi Neviere

\section{To cite this version:}

Xavier Marechal, David Montaigne, Camille Marciniak, Philippe Marchetti, Sidi Mohamed Hassoun, et al.. Doxorubicin-induced cardiac dysfunction is attenuated by cyclosporine treatment in mice through improvements in mitochondrial bioenergetics. Clinical Science, 2011, 121 (9), pp.405-413. 10.1042/CS20110069 . hal-00717079

\author{
HAL Id: hal-00717079 \\ https://hal.science/hal-00717079
}

Submitted on 12 Jul 2012

HAL is a multi-disciplinary open access archive for the deposit and dissemination of scientific research documents, whether they are published or not. The documents may come from teaching and research institutions in France or abroad, or from public or private research centers.
L'archive ouverte pluridisciplinaire $\mathbf{H A L}$, est destinée au dépôt et à la diffusion de documents scientifiques de niveau recherche, publiés ou non, émanant des établissements d'enseignement et de recherche français ou étrangers, des laboratoires publics ou privés. 


\title{
DOXORUBICIN-INDUCED CARDIAC DYSFUNCTION IS ATTENUTED BY CYCLOSPORINE TREATMENT IN MICE THROUGH IMPROVEMENTS IN MITOCHONDRIAL BIOENERGETICS
}

\author{
MARECHAL Xavier \\ MONTAIGNE David \\ MARCINIAK Camille \\ MARCHETTI Philippe \\ HASSOUN Sidi Mohamed \\ BEAUVILLAIN Jean Claude \\ LANCEL Steve \\ NEVIERE Remi
}

Marechal X. and Montaigne D. equally contributed to this work

MX, MD, MC, LS, NR: Department of Physiology (EA 4484), Faculty of Medicine, University North of France

MP and JCB: INSERM U837, JP Aubert Research Center, University North of France

Key words: doxorubicin, heart, biogenesis, mitochondrial transition permeability, energetics Running title page Doxorubicin and cardiac bioenergetics

\section{Corresponding author:}

Dr. Remi NEVIERE, Département de Physiologie, Faculté de Médecine 1, place de Verdun, Lille Cedex 59045 France Tel: (33) 3206269 68; Fax: (33) 3206269 93; email: rneviere@univ-lille2.fr 


\begin{abstract}
We tested whether inhibition of mitochondrial membrane potential dissipation by cyclosporine A would prevent doxorubicin-induced myocardial and mitochondrial dysfunction. Acute and sub-chronic models of doxorubicin exposition were performed in mice with either a single intra peritoneal bolus $(10 \mathrm{mg} / \mathrm{kg}$, i.p. $)$ or one injection of $4 \mathrm{mg} / \mathrm{kg}$ per week during five weeks. Follow-up was 1.5 week and 16 weeks in acute and sub-chronic models, respectively. Mice received either cyclosporine A $(1 \mathrm{mg} / \mathrm{kg}$ i.p. on alternate days $)$ or saline until follow-up. Heart function was evaluated by echocardiography. Mitochondrial measurements included oxygen consumption, membrane potential and externally added calcium-induced mitochondrial permeability transition. Mitochondrial mass was evaluated by transmission electronic microscopy and mitochondrial DNA content. Mitochondrial dynamics was detected as the expression of GTPases involved in mitochondrial fusion and fission. In both acute and chronic models, doxorubicin decreased left ventricular fractional shortening and survival. Heart function and survival were improved by cyclosporine A, but not by tacrolimus (FK506), a cyclosporine derivative with no inhibitory effect on mitochondrial transition pore. In the acute model, doxorubicin exposure was associated with increased mitochondrial DNA content, mitochondrial fragmentation and changes in mitochondrial fusion and fission-related transcripts (increases in Mfn2, OPA1, Fis1, and no changes in Drp1). Cyclosporine A did not alter mitochondrial biogenesis, but prevented mitochondrial fragmentation and partially restored mitochondrial energy-producing capacity. These findings suggest that in vivo cyclosporine A treatment may limit mitochondrial permeability transition pore opening, mitochondrial potential loss and contractile depression in acute and chronic models of cardiac toxicity induced by doxorubicin.
\end{abstract}




\section{INTRODUCTION}

Doxorubicin is an anthracycline antibiotic that is widely used as a chemotherapeutic agent for hematologic malignancies and solid tumors, yet its usefulness is limited by cardiac toxicity [1]. Administration of doxorubicin induces numerous acute cardiac toxic effects, including transient arrhythmias, non-specific electrocardiographic abnormalities, pericarditis, and transient depression of left ventricular function [1,2]. In addition, high cumulative doses are associated with late-onset cardiomyopathy that is refractory to standard treatment. For example, doxorubicin has been reported to induce heart failure in up to $30 \%$ patients receiving $500 \mathrm{mg} / \mathrm{m}^{2}$ or higher cumulative dose with poor clinical outcome[1,2]. Both acute and chronic forms of anthracycline-induced cardiac toxicity have been related to mitochondrial production of reactive oxygen species (ROS) and mitochondrial calcium overload [3-7]. Recent studies suggest that unregulated mitochondrial biogenesis [8,9] and rapid fragmentation of the mitochondrial network [10] are also responsible for the pathogenesis of doxorubicin-induced cardiomyopathy.

Major mechanisms of heart dysfunction induced by oxidative stress, including ROS production, protein damage and mitochondrial injury, have been explored in this model [11]. To ameliorate the cardiotoxic side effects of anthracyclines, several strategies, including limitation of the cumulative dose of anthracyclines, different anthracycline dosage schedules, or different antioxidant agents, have been used, yet providing only modest efficacy [11]. As a different concept, doxorubicin-induced oxidative stress may induce mitochondrial permeability transition, resulting in matrix swelling, uncoupling of the respiratory chain and membrane potential collapse [6]. Interestingly, in vitro preincubation of mitochondria with cyclosporine A, which limits mitochondrial permeability transition by binding to matrix cyclophilin $\mathrm{D}$, reverse the diminished calcium loading capacity of mitochondrial isolated from doxorubicin-treated rats $[3,7]$. In line, mice lacking cyclophilin D, a putative constituent of the mitochondrial permeability transition pore, are protected against doxorubicin cardiotoxicity [12]. We recently showed that non immunosuppressive dose of cyclosporine A can inhibit mitochondrial permeability transition pore opening in both murine and human myocardium preparations, and may largely prevent mitochondrial and contractile dysfunction induced by clinically relevant doses of doxorubicin $[13,14]$. However, information regarding the effects of cyclosporine A on doxorubicin cardiotoxicity in vivo are limited to changes in serum LDH and CPK-MB enzyme levels [15].

The primary objective of this study was to test whether pharmacological inhibition of the mitochondrial transition pore by cyclosporine A would represent a cardioprotective strategy to improve cardiac mitochondrial alterations and myocardial function in both subacute and chronic models of doxorubicin cardiac toxicity performed in young adult (6 to 8week-old) male C57BL/6 mice. Our secondary objective was to test whether cyclosporine A would prevent doxorubicin-induced mitochondrial proliferation and mitochondrial network fragmentation. 


\section{MATERIALS AND METHODS}

\section{Mouse models of doxorubicin cardiotoxicity}

Six- to 8-week-old male C57BL/6 mice (Charles Rivers Laboratories, Lyon, France) were housed for 6 days before manipulation. All experiments were conducted in accordance with the National and European Institutes of Health guidelines for the use of laboratory animals and were approved by the Lille University. The investigation conforms to the Guide for the Care and Use of Laboratory Animals published by the U.S. National Institutes of Health (NIH Publication No. 85-23, revised 1996). Acute (protocol A) and sub-chronic (protocol B) models of doxorubicin exposition were performed in mice with either a single intra peritoneal bolus $(10 \mathrm{mg} / \mathrm{kg}$, i.p.) or one injection of $4 \mathrm{mg} / \mathrm{kg}$ per week during five weeks. Follow-up was 1.5 week and 16 weeks in the acute model and in the sub-chronic, respectively. Control groups received i.p. injection of $0.9 \%$ saline. Control and doxorubicin treated mice were randomized to receive i.p. injection (on alternate days until the end of the study survey )of either 1) vehicle (1\% ethanol in saline); 2) $1 \mathrm{mg} / \mathrm{kg}$ cyclosporine A (CsA); or 3) $0.1 \mathrm{mg} / \mathrm{kg}$ tacrolimus (FK506, a cyclosporine derivative with no inhibitory effect on mitochondrial transition pore).

\section{Animal use and experimental design}

In acute doxorubicin exposure protocol (1.5 week follow-up), three independent sets of animals were used for survival study, echocardiography and mitochondrial evaluation, or ultra structure and biochemistry studies. In sub-chronic doxorubicin exposure protocol (16 week follow-up), two sets of animals were used for either survival or echocardiography study.

\section{Survival studies}

In both acute and sub-chronic studies, survival rate was evaluated twice. An investigator blinded to the identity of the mice performed a 1.5 -week follow-up in the acute doxorubicin exposure protocol (protocol A), and a 16-week follow-up in the sub-chronic doxorubicin exposure protocol (protocol B), in two separate sets of experiments using 20 mice per group.

\section{Echocardiography}

Mice from all treatment groups were anesthetized with isoflurane, and transthoracic echocardiography was performed using Acuson Sequoia C512 system (Mountain View, CA, USA) with a 14-MHz linear transducer. Echocardiographic measurements were taken on Mmode in triplicate for each mouse. In accordance with the American Society of Echocardiography guidelines, the left ventricular fraction shortening (LVFS) was calculated as LVFS = (LVEDD - LVESD)/LVEDD, where LVEDD is left ventricle end diastolic diameter, and LVESD is left ventricle end systolic diameter. All surviving mice were analyzed by echocardiography on day 10 (protocol A) or week 16 (protocol B) and then sacrificed for mitochondrial study (protocol A).

\section{Heart mitochondrial fraction preparation}

Myocardium was excised and placed in isolation buffer A containing (in $\mathrm{mmol} / \mathrm{l}$ ): sucrose 300 , TES 5,EGTA $0.2, \mathrm{pH} 7.2\left(4^{\circ} \mathrm{C}\right)$. The tissue was finely minced and homogenized by the use of a Kontes tissue grinder. After 800- $g$ centrifugation for 5 min, supernatant was centrifuged at $8,800 \mathrm{~g}$ for $5 \mathrm{~min}$. Mitochondrial pellet was resuspended in buffer $\mathrm{A}$ and centrifuged one more time at $8,800 \mathrm{~g}$ for $5 \mathrm{~min}$. Protein concentration was determined according to the Bradford method. Purity and integrity of isolated mitochondria were assessed by measuring specific activities of nicotinamide adenine dinucleotide phosphate-cytochrome c reductase, as an endoplasmic reticulum marker enzyme, and cytochrome c oxidase, as an inner membrane marker enzyme. 


\section{Mitochondrial transmembrane potential and permeability transition}

Isolated mitochondria $(1 \mathrm{mg} / \mathrm{mL}$ proteins) were suspended in buffer $\mathrm{C}$ (in $\mathrm{mM}$ ): sucrose 250; 3-(N-morpholino)propanesulfonic acid MOPS 10; glutamate-Tris 5; malate-Tris 2; Pi-Tris 1; EGTA-Tris $0.02 ; \mathrm{pH} 7.4$ at $25^{\circ} \mathrm{C}$ in a multiport measurement chamber equipped with tetraphenylphosphonium (TPP+)-selective microelectrodes and reference electrodes (WPI, Aston, UK). First, mitochondria were gently stirred for $1.5 \mathrm{~min}$ in buffer C containing $1.5 \mu \mathrm{M}$ TPP+ (Sigma, Saint Quentin Fallavier, France). At the end of the pre-incubation period, $10 \mu \mathrm{mol} / 1 \mathrm{CaCl} 2$ administration was performed every $90 \mathrm{~s}$ with a micro syringe injector adapted to a Micro4 pump controller (UMPII and Micro4, WPI) Each $10-\mu \mathrm{mol} / 1$ $\mathrm{CaCl} 2$ pulse was detected as a peak of extramitochondrial $\mathrm{Ca} 2+$ concentration. The $\mathrm{Ca} 2+$ is then very rapidly taken up by the mitochondria resulting in a return of extramitochondrial $\mathrm{Ca} 2+$ concentration to near baseline level. Following sufficient $\mathrm{Ca} 2+$ loading, so-called calcium retention capacity, extra-mitochondrial $\mathrm{Ca} 2+$ concentration abruptly increases indicating a massive release of $\mathrm{Ca} 2+$ by mitochondria due to permeability transition pore opening. The amount of externally added $\mathrm{Ca} 2+$ necessary to trigger this massive $\mathrm{Ca} 2+$ release is used as an indicator of the susceptibility of mitochondrial permeability transition pore (MPTP) opening to $\mathrm{Ca} 2+$ overload. This amount is expressed in $\mathrm{nmol} / \mathrm{mg}$ protein. To test for involvement of the MPTP opening in the loss of further calcium accumulation, mitochondria were pretreated with $1 \mu \mathrm{M}$ CsA, an inhibitor of the MPTP

Mitochondrial transmembrane potential was estimated by calculating the transmembrane distribution of TPP + . Transmembrane potential $\Delta \Psi \mathrm{m}$ was calculated as $59 \log (\mathrm{v} / \mathrm{V})-59 \log \left(10^{\Delta \mathrm{E} / 59}-1\right)$, where $\mathrm{v}$ is matrix volume $(1.1 \mu \mathrm{l} / \mathrm{mg}$ mitochondrial protein), $\mathrm{V}$ is volume chamber $(1 \mathrm{~mL})$, and $\Delta \mathrm{E}$ is voltage difference before and after calcium induced permeability transition expressed in $\mathrm{mV}$.

\section{Mitochondrial respiratory function in cardiac fibers}

Respiratory function of the total mitochondrial population was studied in saponinskinned fibers prepared from myocardium samples. Briefly, after excision, myocardium was rinsed and placed in the relaxing and biopsy preservation solution BIOPS containing (in $\mathrm{mM}$ ) the following: $\mathrm{CaK}_{2}$ ethyleneglycol-bis-( $(\mathrm{B}$-aminoethyl ether)- $N-N$-tetraacetic acid (EGTA), 2.77; $\mathrm{K}_{2}$ EGTA, 7.2 (free calcium concentration $0.1 \mu \mathrm{M}$ ); $\mathrm{Na}_{2}$ ATP, 5.7; $\mathrm{MgCl}_{2} \cdot 6 \mathrm{H}_{2} \mathrm{O}, 6.6$; taurine, 20; $\mathrm{Na}_{2}$ phosphocreatine, $15 ;$ imidazole, 20; dithiothreitol, $0.5 ; 2-[\mathrm{N}-$ morpholino] ethanesulfonic acid (MES), 50; $\mathrm{pH} 7.1$, as previously described. Isolated fibers were permeabilized for $30 \mathrm{~min}$ with $50 \mu \mathrm{g} / \mathrm{ml}$ of saponin in BIOPS. Then, fibers were washed three times in respiration medium, Mitomed2 (MitoMedium, Oroboros Instruments, Innsbruck, Austria) (see below). All procedures were performed at $4^{\circ} \mathrm{C}$. Respiration was then measured at $25^{\circ} \mathrm{C}$ in a two-chamber respirometer (Oxygraph-2k; Oroboros Instruments) : five to $10 \mathrm{mg}$ of fiber bundles were placed in a chamber containing $2 \mathrm{ml}$ of Mitomed2 (in $\mathrm{mM}$ : $\mathrm{Na}_{2}$ EDTA, 0.5; $\mathrm{MgCl}_{2} \cdot 6 \mathrm{H}_{2} \mathrm{O}, 5 ; \mathrm{KH}_{2} \mathrm{PO}_{4}, 10$; mannitol, 110; KCl, 60; Tris, 60; $\mathrm{pH}$ 7.4). The $\mathrm{O}_{2}$ solubility of this medium was taken as $11.3 \mu \mathrm{M} / \mathrm{kPa}$.

Respiration rates of 5-10 $\mathrm{mg}$ of skinned fibers were then measured at $25^{\circ} \mathrm{C}$ in a twochamber respirometer (Oroboros $\mathrm{O}_{2} \mathrm{k}$ oxygraph, Innsbruck, Austria) containing $2 \mathrm{~mL}$ of Mitomed2 (in mM: $\mathrm{Na}_{2}$ EDTA $0.5, \mathrm{MgCl}_{2} \cdot 6 \mathrm{H}_{2} \mathrm{O} 5, \mathrm{KH}_{2} \mathrm{PO}_{4} 10$, mannitol 110, $\mathrm{KCl}$ 60, Tris $60 ; \mathrm{pH} 7.4$ ) with or without substrates. State 4 respiration (without ADP) was first determined with glutamate $(5 \mathrm{mM})+$ malate $(2 \mathrm{mM})$. State 3 respiration was then achieved by adding ADP $(2.5 \mathrm{mM})$ to the preparation. Respiratory control ratio (RCR) was determined by calculating the ratio between ADP-stimulated respiration over state 4 respiration (without ADP) with glutamate and malate as substrate. At the end of the respiratory protocol, the addition of cytochrome c $(10 \mu \mathrm{M})$ was used to check mitochondrial outer membrane permeability. Rates of respiration are given in picomoles $\mathrm{O}_{2}$ per second per mg wet weight. 


\section{Heart ultrastructure}

Mouse hearts were examined for morphometric analysis of mitochondrial volume density and fragmentation. One block of tissue was isolated from the left ventricular free wall of each heart. These blocks were fixed with glutaraldehyde, embedded and oriented for crosssectional views. Thin sections were stained with uranyl acetate and lead citrate, and examined using a Phillips CM10 transmission electron microscope (Phillips, Eindhoven, The Netherlands). An individual who was blinded to the treatment groups performed the electron microscopic examination of sections, photographed ten representative prints per block, made prints at standardized magnification $(12,300 \mathrm{x})$ and performed point counting using a standard grid overlay. The grid was $18 \times 18 \mathrm{~cm}$ with lines spaced by $1 \mathrm{~cm}$. Each of the grid intersects was scored by the blinded individual to determine if the intersect fell upon a mitochondrial profile or was outside of mitochondria. The ratio (number of grid intersects superimposing mitochondrial profiles / number of total possible intersects) represents the mitochondrial volume density, which was averaged for each heart. In longitunally-sectioned cardiomyocytes, lengths of individual mitochondria were measured using Metamorph softxare. For each heart, approximately 1000 mitochondria were measured on ten representative prints $(12,300 \mathrm{x})$ to determine the percentage distribution of mitochondria with various lengths: $0-1.5 \mu \mathrm{m} ; 1.5-2 \mu \mathrm{m}$ (sarcomere length), $\geq 2 \mu \mathrm{m}$. An index of mitochondrial fragmentation was the percent of mitochondria of more than $1.5 \mu \mathrm{m}$.

\section{Mitochondrial DNA (mtDNA) copy number and real-time PCR}

Total DNA (genomic + mitochondrial) was extracted from heart tissue using QIAamp DNA Mini Kit (Qiagen, France). The relative mtDNA copy number was measured by PCR and corrected by simultaneous measurement of the nuclear DNA. The forward and reverse primers for mtDNA which are complementary to the sequence of the mouse mitochondrial cytochrome c oxidase subunit II gene, were 5'-AACCATAGGGCACCAATGATAC-3' and 5'-GGATGGCATCAGTTTTAAGTCC-3'. 5'-CGGCGACGACCCATTCGAAC-3' and 5'GAATCGAACCCTGATTCCCCGTC-3', sequences complementary to the $18 \mathrm{~S}$ gene, were the primers used for the detection of nuclear DNA. Quantitative PCR was performed on an Eppendorf Realplex S2 (Eppendorf, Germany) using Mesa Blue Mix (Eurogentec, France). Results are expressed as $2-\Delta \Delta \mathrm{Ct}$.

\section{Real-time PCR}

Total RNAs from flash frozen heart tissue were extracted using TriZol reagent (Invitrogen) and purified with the PureLink Micro-to-Midi total RNA purification kit (Invitrogen). Total RNAs (1 $\mu \mathrm{g})$ were reverse-transcribed using transcriptor first strand cDNA synthesis kit (Roche) according manufacturer's instructions. Real-time RT-PCR was performed using an Eppendorf Realplex S2 (Eppendorf, Germany) and Mesa Blue qPCR Master Mix Plus for SYBR assay (Eurogentec, France). Primers for mouse PGC-1 $\alpha$, Mfn2, OPA1, Drp1, Fis1 (Table 1) were from Invitrogen. $\beta$-actin was used as an internal control. Realplex Software was used to quantify differences in gene expression. Results are expressed as $2-\Delta \Delta \mathrm{Ct}$.

\section{Statistics}

Results were analyzed with the SPSS for Windows software, version 11.0.1 (SPSS France, Paris-la-Défense, France). Data represent means \pm SEM and were analyzed by analysis of variance procedures. When a significant difference was found, we identified specific differences between groups with a sequentially rejective Bonferroni procedure. After application of the Bonferroni correction, $\mathrm{p}<0.05$ was taken as a level of statistical significance. Survival was evaluated with Fisher exact test. 


\section{RESULTS}

\section{Cyclosporine treatment improved survival in doxorubicin-treated mice}

Doxorubicin toxicity was evaluated in vivo using two different protocols of drug administration, i.e. protocols A and B (see Methods for details). Mice mortality was observed only in mice that received doxorubicin treatment. Doxorubicin-treated mice began to die between 4 and 5 days of follow-up in protocol A, and between 6 and 8 weeks in protocol $\mathrm{B}$. Mortality rate was $55 \%$ at day 8 in protocol $\mathrm{A}$ and then remained stable till day 10 . In protocol B, a maximal mortality rate of $60 \%$ was reached in the doxorubicin treated group after 13 weeks. In both protocols A and B, CsA $(1 \mathrm{mg} / \mathrm{kg} / 48 \mathrm{~h})$ significantly improved survival rate after administration of doxorubicin $(25 \%$ and $40 \%$ mortality rate in protocol $\mathrm{A}$ and $\mathrm{B}$, respectively; $\mathrm{p}<0.05$ compared with controls), whereas FK506 exerted deleterious effects on mortality rate (data not shown) in doxorubicin-treated mice.

\section{Cyclosporine prevented doxorubicin-induced myocardial contractile dysfunction}

At baseline, heart rate and LVFS were similar among the 6 groups of mice. At day 10 of protocol A, doxorubicin induced a significant left ventricular systolic dysfunction in mice compared with control group $(\mathrm{p}<0.05)$ (Table 2). Treatment with CsA reduced doxorubicin mediated myocardial dysfunction. FK506 exerted no effect on left ventricular function of doxorubicin-exposed mice (Table 2). In protocol B, similar results were observed on echocardiographic study at week 16 with protective action of CsA against doxorubicin-related contractile dysfunction (Table 2).

\section{Cyclosporine prevented doxorubicin-induced cardiac mitochondrial dysfunction}

Respiration measurements were started immediately after preparation of permeabilized cardiac fibers. Addition of cytochrome c $(10 \mu \mathrm{M})$ had no significantly effects on state 3 respiration (data not shown), suggesting that procedures used to prepare cardiac fibers did not damage mitochondrial outer membrane. In this setting, doxorubicin altered oxidative phosphorylation respiration in mouse model of cardiotoxicity, as reflected by reduced respiratory control ratio (RCR) (Table 3). Reduction of RCR induced by doxorubicin was the result of both reduced $\mathrm{ADP}$-stimulated respiration and increased state 4 respiration (without ADP) with glutamate and malate as substrates in mouse sub-acute model. In this context, administration of CsA largely prevented doxorubicin-induced deterioration of mitochondrial respiration parameters at 10 days after doxorubicin administration in protocol A (Table 3). Doxorubicin cardiotoxicity was accompanied by mitochondrial membrane potential dissipation (Table 3 ), which was prevented by cyclosporine. The amount of $\mathrm{Ca} 2+$ required to open the transition pore averaged $220 \pm 18 \mu \mathrm{mol} / \mathrm{L}$ in isolated mitochondria from control mice, which corresponded to $220 \mathrm{nmol} / \mathrm{mg}$ of mitochondrial protein. A similar amount of calcium was required to open the pore in CsA-treated contol groups. The calcium overload require to induce mitochondrial permeability transition was significantly reduced in doxorubicin group, averaging $93 \pm 25 \mu \mathrm{mol} / \mathrm{L}$ in mice mitochondria. Whereas FK506 had no effects, CsA in vivo treatment significantly increased the calcium amount required for pore opening after doxorubicin exposure (Table 3). In select experimental runs, in vitro preincubation with CsA, a known inhibitor of the mitochondrial permeability transition (MPTP), delayed calcium-induced calcium release in both control and doxorubicin mitochondria (data not shown). The findings that in vitro CsA increased calcium uptake capacity suggested that the loss of ability to take up further calcium correlated with MPTPdependent process.

Effects of cyclosporine on cardiac mitochondrial biogenesis and network dynamic in doxorubicin-treated mice

At day 10 post-doxorubicin, mitochondrial ultrastructure analyses indicated increased mitochondrial density and decreased section areas of individual mitochondria (Figure 1), 
suggesting increased population of mitochondria and reduced mitochondrial size (Table 4). Compared with controls, percentage of mitochondrial size less than $1.5 \mu \mathrm{m}$ was increased, whereas percentage of elongated mitochondria (mitochondria of more than $1.5 \mu \mathrm{m}$ ) was reduced in doxorubicin-treated mice, suggesting increased mitochondrial fragmentation (Table 4). Mitochondrial biogenesis profile in real time quantitative PCR indicated a 2-fold increase in mitochondrial DNA content (Figure 2) and a 3-fold increase in PGC-1 $\alpha$ in doxorubicin-treated mice (Figure 2). Mitochondrial fusion and fission profile in real time quantitative PCR included a 4-fold increase in Mfn2, a 2-fold increase in OPA1, a 2-fold increase in Fis1, whereas Drp1 was unchanged in doxorubicin-treated mice (Figure 3).

Increases in mitochondrial DNA content increase and mitochondrial biogenesis induced by doxorubicin was not prevented by cyclosporine A treatment (Figure 2). Cyclosporine A largely prevented alterations in mitochondrial fusion/fusion balance (Figure 3) and ultrastructural changes (Table 4) observed in doxorubicin-treated mice. Normalization of mitochondrial fusion/fission balance together with maintained mitochondrial biogenesis signal induced by cyclosporine A was associated with major improvements in respiratory capacity (Table 3). Tacrolimus (FK506), a calcineurin inhibitor that does not interact with mitochondrial targets, had no effects on mitochondrial respiration (Table 3), myocardial contractile performance and survival in doxorubicin-treated mice.

\section{DISCUSSION}

Our study reveals the important findings that cyclosporine A, a potent inhibitor of mitochondrial transition pore, protected mice against sub-acute and chronic doxorubicin cardiotoxicity. The cyclosporine derivative FK506, having no effects on mitochondria, exerted no such beneficial effects, suggesting that cardioprotection associated with cyclosporine A was related to its inhibitory property on the mitochondrial permeability transition. Treatment with cyclosporine A was accompanied by preservation of cardiac bioenergetics and mitochondrial network organization, which could be involved in the observed cardioprotection against doxorubicin.

Doxorubicin increases the susceptibility of mitochondria to oxidative stress and $\mathrm{Ca}^{2+}$ induced mitochondrial permeability transition, which contributes to a mitochondrial bioenergetic failure [6]. Effects of doxorubicin administration in vivo on mitochondrial respiration can be restored by adding cyclosporine A in vitro, implicating mitochondrial permeability transition as a direct causative factor of doxorubicin on mitochondrial bioenergetics [7]. Similarly, the findings of the present study suggest that in vivo treatment with low doses of cyclosporin A may limit mitochondrial permeability transition pore opening, mitochondrial potential loss and contractile depression in acute and chronic models of cardiac toxicity induced by doxorubicin [13,14]. Previous studies on the effects of cyclosporine A on cardiotoxicity have yielded conflicting results. Mice pretreated with high concentrations of cyclosporine $(12.5$ or $25 \mathrm{mg} / \mathrm{kg}$ ) are more vulnerable to doxorubicin toxicity $[16,17]$. Deleterious effects of high doses of cyclosporine have been related to inhibition of the membrane transport P-glycoprotein, which acts as an energy-dependent pump able to increase doxorubicin cellular efflux and reduce its intracellular retention [16,17]. In contrast, low doses of cyclosporine $(0.5-2 \mathrm{mg} / \mathrm{kg})$, which have no inhibitory effects on P-glycoprotein, may decrease doxorubicin-induced myocardial damage, i.e., elevation of serum LDH and CPK-MB enzymes [15]. Our results are consistent with studies showing that low dose cyclosporine can protect the myocardium in the context of cardiac injury induced by doxorubicin. Interestingly, beneficial effects of mitochondrial permeability transition pore inhibitor cyclosporine A have been shown to generate a similar protection in acute myocardial infarction patients [18]. Mechanisms by which cyclosporine A can prevent doxorubicin- 
induced cardiotoxicity involve alteration of mitochondrial transmembrane potential through its inhibitory property on the mitochondrial permeability transition $[3,7,13,14]$. Yet significant and consistent with our previous studies [13,14], the decrease in membrane potential observed in mitochondria prepared from doxorubicin-treated mice could not be sufficient to directly compromise ATP synthesis. For example, it has been proposed that the ATP synthesis kinetics is a sigmoid function of membrane potential that reaches saturation at approximately (minus) 100 mvolts [19].

One possible explanation for the protective effects of cyclosporine A may be associated with the preservation of a healthy mitochondrial network thank to mitochondrial dynamics. Mitochondrial dynamics refers to repetitive cycles of fusion and fission between mitochondria that are controlled by GTPases involved in fusion, OPA1 and Mfn2, and the fission protein Fis1 [20,21]. These opposing processes determine the architecture of the entire mitochondrial population and influence mitochondrial functions, including respiration and calcium buffering [22-24]. Mitochondrial transmembrane potential has been critically implicated the regulation of mitochondrial dynamics, with depolarized mitochondria being the substrate for mitochondrial autophagy [22,23]. In our study, mitochondrial dynamics was altered, as doxorubicin increased mRNA expression of GTPases involved in mitochondrial fusion, OPA1 and Mfn2, and proteins involved in mitochondrial fission, such as Fis1. These new information are important as derangements of mitochondrial dynamics play a critical role in the failing heart [25]. The findings that cyclosporine A normalized RNA expression of GTPases involved in fusion, OPA1 and Mfn2, and the fission protein Fis1, suggest that changes in mitochondrial membrane potential can modulate processes that govern mitochondrial dynamics. In line, recent findings in embryonic cardiac cell line have shown that cyclosporine A blocked dissipation of membrane potential and changes in OPA1 expression that occurred during simulated ischemia in H9c2 cell lines [25]. Overall, it may be proposed that dissipation of mitochondrial membrane potential associated with doxorubicin cardiotoxicity may induce mitochondrial network fragmentation and subsequent mitochondrial dysfunction. In this context, stabilization of membrane potential by cyclosporine A would result in mitochondrial bioenergetics preservation and improved cardiac function.

Alternative explanation for the beneficial effects of cyclosporine A on doxorubicininduced mitochondrial dysfunction may be changes in heart mitochondrial biogenesis. Previous studies reported that doxorubicin may elicit mitochondrial DNA polymerase activation, DNA repair and replication, and mitochondrial proliferation in response to heart oxidative mitochondrial damage [26,27]. In sharp contrast, mitochondrial biogenesis inhibition has also been found in acute models of doxorubicin cardiotoxicity [9,28]. In our model, we observed that doxorubicin administration was accompanied with heart mitochondrial proliferation and increased DNA content, suggesting mitochondrial biogenesis stimulation. In doxorubicin-treated mice heart, cyclosporine A did not altered mitochondrial biogenesis, DNA content and PGC- $1 \alpha$ protein expression even though the mitochondrial content, i.e., density and size, was normalized. Interpretation of these conflicting results is not readily evident as the effects of cyclosporine A on mitochondrial biogenesis are largely unknown. Calcineurin has been shown to increase PGC-1 $\alpha$ gene transcription, and overexpression of constitutively active calcineurin in skeletal muscle of transgenic mice results in increased expression of PGC-1 $\alpha$ [29,30]. Furthermore, expression of constitutively active calcineurin in cardiac myocytes has been shown to result in increased expression of PGC-1 $\alpha$ and the induction of a wide range of genes involved in mitochondrial energy metabolism [30]. In this context, inhibition of calcineurin with cyclosporine A did not prevent increases in PGC-1 $\alpha$ and mitochondrial proteins [31]. These results are consistent with the 
absence of effects of cyclosporine A on increased DNA content and PGC-1 $\alpha$ protein expression in our model.

Overall, doxorubicin is an anthracycline antibiotic that is widely used in the clinical setting as a major chemotherapeutic agent, yet its usefulness is limited by cardiac toxicity. Unfortunately, several strategies aimed to ameliorate this cardiotoxicity (i.e., limitation of the cumulative dose of anthracyclines, different anthracycline dosage schedules, use of different antioxidant agents) have providing only modest efficacy. As a different concept, our studies suggest that, in animal and human myocardium, low dose of cyclosporine A can prevented doxorubicin-induced mitochondrial and myocardial contractile dysfunction through its inhibitory effects on mitochondrial permeability transition $[13,14]$. Such beneficial effects of cyclosporine A have been recently demonstrated in patients suffering from acute myocardial infarction with promising results on infarct size [18].

In conclusion, our study suggests that mitochondrial membrane potential stabilization with cyclosporine A prevents doxorubicin-induced heart contractile dysfunction through, at least part, modulation of mitochondrial bioenergetics. 


\section{Author's contributions:}

Mr. Marechal X. Montaigne D. and Marciniak C. were responsible for data collection and analyzed the data. Authors reviewed the final manuscript.

Mr. Beauvillain JC was responsible for transmission electronic microscopy and review of the manuscript.

Mr. Lancel S. was responsible for statistical analysis and reviewing the manuscript.

Mr. Hassoun SM. Was responsible for reviewing the manuscript.

Mr Neviere R. was responsible for data collection, analyzed the data and wrote the manuscript.

\section{Acknowledgements:}

We thank Mrs Loyens Anne for technical assistance and expertise in electronic microscopy studies

Funding:

This work was supported by grants from EA 4484 University of Lille 2, France 


\section{REFERENCES}

1. Minotti, G., Menna, P., Salvatorelli, E., Cairo, G., and Gianni, L. (2004) Anthracyclines: molecular advances and pharmacologic developments in antitumor activity and cardiotoxicity. Pharmacol. Rev. 56, 185-229

2. Ferreira, A.L., Matsubara, L.S., and Matsubara, B.B. (2008) Anthracycline-induced cardiotoxicity. Cardiovasc. Hematol. Agents Med. Chem. 6, 278-281

3. Solem, L.E., Heller, L.J., and Wallace, K.B. (1996) Dose-dependent increase in sensitivity to calcium-induced mitochondrial dysfunction and cardiomyocyte cell injury by doxorubicin. J. Mol Cell Cardiol 28, 1023-32

4. Cardoso, S., Santos, R.X., Carvalho, C., Correia, S., Pereira, G.C., Oliveira PJ, Santos, M.S., Proenca, T. and Moreira, P.I. (2008) Doxorubicin increases the susceptibility of brain mitochondria to $\mathrm{Ca}(2+)$-induced permeability transition and oxidative damage. Free Radic. Biol. Med. 45, 1395-1402

5. Oliveira, P.J., Goncalves, L., Monteiro, P., Providencia, L.A., and Moreno, A.J. (2005) Are the antioxidant properties of carvedilol important for the protection of cardiac mitochondria? Curr. Vasc. Pharmacol. 3, 147-158

6. Wallace, K.B. (2007) Adriamycin-induced interference with cardiac mitochondrial calcium homeostasis. Cardiovasc. Toxicol. 7, 101-107

7. Zhou, S., Starkov, A., Froberg, M.K., Leino, R.L., and Wallace, K.B. (2001) Cumulative and irreversible cardiac mitochondrial dysfunction induced by doxorubicin. Cancer Res. 61, 771-777

8. Palmeira, C.M., Serrano, J., Kuehl, D.W., and Wallace, K.B. (1997) Preferential oxidation of cardiac mitochondrial DNA following acute intoxication with doxorubicin. Biochim. Biophys. Acta 1321, 101-106

9. Suliman, H.B., Carraway, M.S., Ali, A.S., Reynolds, C.M., Welty-Wolf, K.E., and Piantadosi, C.A. (2007) The CO/HO system reverses inhibition of mitochondrial biogenesis and prevents murine doxorubicin cardiomyopathy. J. Clin. Invest. 117, 3730-3741

10. Parra, V., Eisner, V., Chiong, M., Criollo, A., Moraga, F., Garcia, A., Hartel, S., Jaimovich, E., Zorzano, A., Hidalgo, C., and Lavandero S (2008) Changes in mitochondrial dynamics during ceramide-induced cardiomyocyte early apoptosis. Cardiovasc. Res. 77, 387-397

11. Simunek, T., Sterba, M., Popelova, O., Adamcova, M., Hrdina, R., and Gersl, V. (2009) Anthracycline-induced cardiotoxicity: overview of studies examining the roles of oxidative stress and free cellular iron. Pharmacol. Rep. 61, 154-171

12. Nakayama, H., Chen, X., Baines, C.P., Klevitsky, R., Zhang, X., Zhang, H., Jaleel, N., Chua, B.H., Hewett, T.E., Robbins, J., Houser, S.R., and Molkentin, J.D. (2007) Calcium and mitochondrial-dependent cardiomyocyte necrosis as a primary mediator of heart failure. J. Clin. Invest. 117, 2431-44

13. Montaigne, D., Marechal, X., Baccouch, R., Modine, T., Preau, S., Zannis, K., Marchetti, P., Lancel, S. and Neviere R (2010) Stabilization of mitochondrial membrane potential prevents doxorubicin-induced cardiotoxicity in isolated rat heart. Toxicol. Appl. Pharmacol. 244, 300-307

14. Montaigne, D., Marechal, X., Preau, S., Baccouch, R., Modine, T., Fayad, G., Lancel, S., and Neviere, R. (2011) Doxorubicin induces mitochondrial permeability transition and contractile dysfunction in the human myocardium. Mitochondrion 211,22-26

15. Al-Nasser, I.A. (1998) In vivo prevention of adriamycin cardiotoxicity by cyclosporin A or FK506. Toxicology 131, 175-181 
16. Bellamy, W.T., Peng, Y.M., Odeleye, A., Ellsworth, L., Xu, M.J., Rogan, T.M. and Weinstein, R.S. (1995) Cardiotoxicity in the SCID mouse following administration of doxorubicin and cyclosporin A. Anticancer. Drugs 6, 736-743

17. Colombo, T., Zucchetti, M., and D'Incalci, M. (1994) Cyclosporin A markedly changes the distribution of doxorubicin in mice and rats. J. Pharmacol. Exp Ther. 269, 22-27

18. Piot, C., Croisille, P., Staat, P., Thibault, H., Rioufol, G., Mewton, N., Elbelghiti, R., Cung, T.T., Bonnefoy, E., Angoulvant, D., Macia, C., Raczka, F., Sportouch, C., Gahide, G., Finet, G., Andre-Fouet, X., Revel, D., Kirkorian, G., Monassier, J.P., Derumeaux, G. and Ovize M (2008) Effect of cyclosporine on reperfusion injury in acute myocardial infarction. N. Engl. J. Med. 359, 473-481

19. Dimroth, P., Kaim, G., and Matthey, U. (2000) Crucial role of the membrane potential for ATP synthesis by F(1)F(o) ATP synthases. J. Exp. Biol. 203, 51-59.

20. Kane, L.A., and Youle, R.J. (2010) Mitochondrial fission and fusion and their roles in the heart. J. Mol. Med. 88, 971-999

21. Ong, S.B., and Hausenloy, D.J. (2010) Mitochondrial morphology and cardiovascular disease. Cardiovasc. Res. 88, 16-29

22. Ong, S.B., Subrayan, S., Lim, S.Y., Yellon, D.M., Davidson, S.M., and Hausenloy, D.J. (2010) Inhibiting mitochondrial fission protects the heart against ischemia/reperfusion injury. Circulation 121, 2012-2022

23. Benard, G., and Rossignol, R. (2008) Ultrastructure of the mitochondrion and its bearing on function and bioenergetics. Antioxid. Redox Signal. 10, 1313-1342

24. Sauvanet, C., Duvezin-Caubet, S., di Rago, J.P., Rojo, M. (2010) Energetic requirements and bioenergetic modulation of mitochondrial morphology and dynamics. Semin. Cell. Dev. Biol. 21, 558-565

25. Chen, L., Gong, Q., Stice, J.P., and Knowlton, A.A. (2009) Mitochondrial OPA1, apoptosis, and heart failure. Cardiovasc. Res. 84, 91-99

26. Kluza, J., Marchetti, P., Gallego, M.A., Lancel, S., Fournier, C. Loyens, A., Beauvillain, J.C., and Bailly C (2004) Mitochondrial proliferation during apoptosis induced by anticancer agents: effects of doxorubicin and mitoxantrone on cancer and cardiac cells. Oncogene 23, 7018-7030

27. Ogihara, M., Tanno, M., Izumiyama, N., Nakamura, H., and Taguchi, T. (2002) Increase in DNA polymerase gamma in the hearts of adriamycin-administered rats. Exp. Mol. Pathol. 73, 234-241

28. Miyagawa, K., Emoto, N., Widyantoro, B., Nakayama, K., Yagi, K., Rikitake, Y., Suzuki, T., and Hirata, K. (2010) Attenuation of Doxorubicin-induced cardiomyopathy by endothelin-converting enzyme-1 ablation through prevention of mitochondrial biogenesis impairment. Hypertension 55, 738-746

29. Garcia-Roves, P.M., Huss, J., and Holloszy, JO. (2006) Role of calcineurin in exercise-induced mitochondrial biogenesis. Am. J. Physiol. 290, E1172-9

30. Handschin C, Rhee J, Lin J, Tarr PT, and Spiegelman BM. An autoregulatory loop controls peroxisome proliferator-activated receptor gamma coactivator 1alpha expression in muscle. Proc Natl Acad Sci USA 100: 7111-7116, 2003.

31. Schaeffer PJ, Wende AR, Magee CJ, Neilson JR, Leone TC, Chen F, and Kelly DP. Calcineurin and calcium/calmoduln-dependent protein kinase activate distinct metabolic gene regulatory programs in cardiac muscle. J Biol Chem 279: 3959339603, 


\section{FIGURE LEGENDS}

\section{Figure 1: Heart ultrastructure}

Myocardium electron micrographs (same magnification, bar $=1 \mu \mathrm{m}$ ) in control (left) with normal myofibrillary and mitochondria structures and doxorubicin (middle) treated mice. The mitochondria in the cardiac ventricles of doxorubicin-treated mice were more numerous and often significantly larger than control mouse hearts. In some myocytes, the marked mitochondrial proliferation appeared to have replaced the sarcomeric assembly. Righ panel is electron micrograph of Csa doxorubicin treated heart.

\section{Figure 2: Mitochondrial biogenesis assessment}

Effects of doxorubicin (doxo) on mitochondrial DNA copy number (panel A) and PGC-1 $\alpha$ RNA expression (panel B). Results are expressed as mean \pm SEM ( $\mathrm{n}=8$ mice in each group). * $\mathrm{P}<0.05$ compared with matched control. CsA is cyclosporine A. See materials and methods for doxorubicin (doxo) and cyclosporine A (CsA) treatment groups.

\section{Figure 3: Mitochondrial dynamics profile}

Effects of doxorubicin (doxo) on relative RNA levels of Mfn2 (panel A), OPA1 (panel B), Drp1 (panel C) and Fis1 (panel D). Results are expressed as mean \pm SEM ( $n=8$ mice in each group). ${ }^{*} \mathrm{P}<0.05$ compared with matched control. CsA is cyclosporine A. See materials and methods for doxorubicin (doxo) and cyclosporine A (CsA) treatment groups. CsA had no effects in control (data not shown). 
Table 1: Gene accession numbers and primer sequences

\begin{tabular}{|c|c|c|c|}
\hline Accession Number & Gene & \multicolumn{2}{|c|}{ Primer sequence } \\
\hline \multirow[t]{2}{*}{ NM_008904 } & PGC-1 $\alpha$ & Forward & CGGAAATCATATCCAACCAG \\
\hline & & Reverse & TGAGGACCGCTAGCAAGTTTG \\
\hline \multirow[t]{2}{*}{ NM_133201 } & Mfn2 & Forward & GGGGCCTACATCCAAGAGAG \\
\hline & & Reverse & GCAGAACTTTGTCCCAGAGC \\
\hline \multirow[t]{2}{*}{ NM_133752 } & OPA1 & Forward & GATGACACGCTCTCCAGTGAAC \\
\hline & & Reverse & CTCGGGGCTAACAGTACAACC \\
\hline \multirow[t]{2}{*}{ NM_152816 } & Drp1 & Forward & CGGTTCCCTAAACTTCACGA \\
\hline & & Reverse & GCACCATTTCATTTGTCACG \\
\hline \multirow[t]{2}{*}{ NM_025562 } & Fis 1 & Forward & CCGGCTCAAGGAATATGAAA \\
\hline & & Reverse & CCATGCCTACCAGTCCATCT \\
\hline \multirow[t]{2}{*}{ NM_007393 } & $\beta$-actin & Forward & AGCTGCCTGACGGCCAGGTC \\
\hline & & Reverse & GCTCAGGAGGAGCAATGATC \\
\hline
\end{tabular}


Table 2: Left ventricular fraction shortening assessed by echocardiography in mice
LVFS
At 1.5 week doxorubicin
At 16 week doxorubicin

$\begin{array}{lcc}\text { Control } & 0.40 \pm 0.02 & 0.42 \pm 0.02 \\ \text { CsA } & 0.40 \pm 0.03 & 0.38 \pm 0.03 \\ \text { FK506 } & 0.43 \pm 0.05 & 0.40 \pm 0.02 \\ \text { Doxo } & 0.31 \pm 0.03^{*} & 0.28 \pm 0.04^{*} \\ \text { Doxo CsA } & 0.39 \pm 0.04 & 0.35 \pm 0.03 \\ \text { Doxo FK506 } & 0.32 \pm 0.05^{*} & 0.29 \pm 0.05^{*}\end{array}$

Echocardiographic measurements were performed in triplicate for each mouse. Left ventricular fraction shortening (LVFS) was calculated in accordance with the American Society of Echocardiography guidelines. See materials and methods for doxorubicin (doxo), ciclosporine A (CsA) and tacrolimus (FK506) treatment groups. Data are mean \pm SEM. Results were analyzed with one-way ANOVA and Bonferroni's multiple comparison post hoc adjustment ( $\mathrm{n}=12$ in each group; * indicates $\mathrm{p}<0.05$ vs control). 
Table 3: Mitochondrial functional studies in cardiac fibers and isolated mitochondria

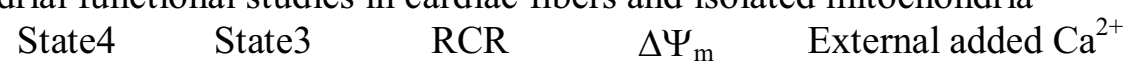

$\begin{array}{cccccc}\text { control } & 53 \pm 2 & 213 \pm 9 & 3.8 \pm 0.5 & 205 \pm 5 & 220 \pm 18 \\ \text { CsA } & 54 \pm 4 & 231 \pm 5 & 4.0 \pm 0.2 & 210 \pm 3 & 225 \pm 16 \\ \text { FK506 } & 50 \pm 4 & 205 \pm 6 & 4.0 \pm 0.6 & 200 \pm 4 & 200 \pm 9 \\ \text { Doxo } & 65 \pm 5^{*} & 138 \pm 8^{*} & 2.3 \pm 0.5^{*} & 183 \pm 7^{*} & 93 \pm 25^{*} \\ \text { Doxo CsA } & 52 \pm 3 & 195 \pm 8^{*} \dagger & 3.4 \pm 0.6^{*} \dagger & 206 \pm 6 & 179 \pm 13^{*} \dagger \\ \text { Doxo FK506 } & 70 \pm 5^{*} & 120 \pm 8^{*} & 2.31 \pm 0.7^{*} & 173 \pm 5^{*} & 83 \pm 25^{*}\end{array}$

See materials and methods for doxorubicin (Doxo), cyclosporine A (CsA) and tacrolimus (FK506) treatment groups. Rates of respiration in cardiac fibers are given in picomoles O2 per second per mg wet weight ( $\mathrm{pmol} / \mathrm{O} / \mathrm{sec} / \mathrm{mg}$ ). State 4 respiration rate is determined with glutamate $(5 \mathrm{mM})+$ malate $(2 \mathrm{mM})$ without ADP. State 3 respiration rate is determined in the presence of ADP $(2.5 \mathrm{mM})$ with mitochondrial substrates added to the preparation. Respiratory control ratio (RCR) is the ratio between state 3 respiration rate over state 4 . Assessment of mitochondrial membrane potential $(\Delta \psi \mathrm{m}$, mvolts) and $\mathrm{Ca} 2+$ pulse studies are performed in isolated mitochondria. External added $\mathrm{Ca} 2+(\mathrm{nmol} / \mathrm{mg}$ protein) represents the amount of externally added $\mathrm{Ca} 2+$ necessary to trigger the massive $\mathrm{Ca} 2+$ release, an indicator of mitochondrial permeability transition. Data are mean \pm SEM. Results were analyzed with one-way ANOVA and Bonferroni's multiple comparison post hoc adjustment ( $\mathrm{n}=12$ in each group; * indicates $\mathrm{p}<0.05$ vs control; $\dagger$ indicates $\mathrm{p}<0.05$ doxo vs doxo CsA). 
Table 4: Mitochondrial ultra structure analyses in mice heart

$\begin{array}{ccccc} & \begin{array}{c}\text { Mitochondria } \\ \text { density }\end{array} & \begin{array}{c}\text { Mitochondrial } \\ \text { size }\end{array} & \begin{array}{c}\text { Elongated } \\ \text { mitochondria }\end{array} & \begin{array}{c}\text { Fragmented } \\ \text { mitochondria }\end{array} \\ \text { control } & 0.38 \pm 0.01 & 1.12 \pm 0.04 & 20.2 \pm 2.4 & 79.8 \pm 2.4 \\ \text { CsA } & 0.39 \pm 0.01 & 1.09 \pm 0.02 & 20.3 \pm 0.6 & 79.4 \pm 0.6 \\ \text { Doxo } & 0.47 \pm 0.01^{*} & 0.94 \pm 0.06^{*} & 7.7 \pm 1.6^{*} & 92.3 \pm 1.7^{*} \\ \text { Doxo CsA } & 0.40 \pm 0.01 & 1.10 \pm 0.02 & 16.8 \pm 1.4^{*} \dagger & 83.2 \pm 1.4^{*} \dagger\end{array}$

See materials and methods for doxorubicin (Doxo) and cyclosporine A (CsA) treatment groups. Mitochondria volume density is expressed in $\mu \mathrm{m} 3$ of mitochondria per $\mu \mathrm{m} 3$ total tissue volume. Mitochondrial size is the mean of 1000 individual mitochondrial crosssectional diameter and expressed in $\mu \mathrm{m}$. Elongated mitochondria are mitochondria of more than $1.5 \mu \mathrm{m}$ and expressed in percent of the mitochondria number per area. Percentage of mitochondria of less than $1.5 \mu \mathrm{m}$ was used as an index of mitochondrial fragmentation. Data are mean \pm SEM $(n=8$ hearts in each group). Results were analyzed with one-way ANOVA and Bonferroni's multiple comparison post hoc adjustment $(*$ indicates $\mathrm{p}<0.05$ vs control; †indicates $\mathrm{p}<0.05$ doxo vs doxo CsA). 


\section{A}

\section{mitochondrial DNA}

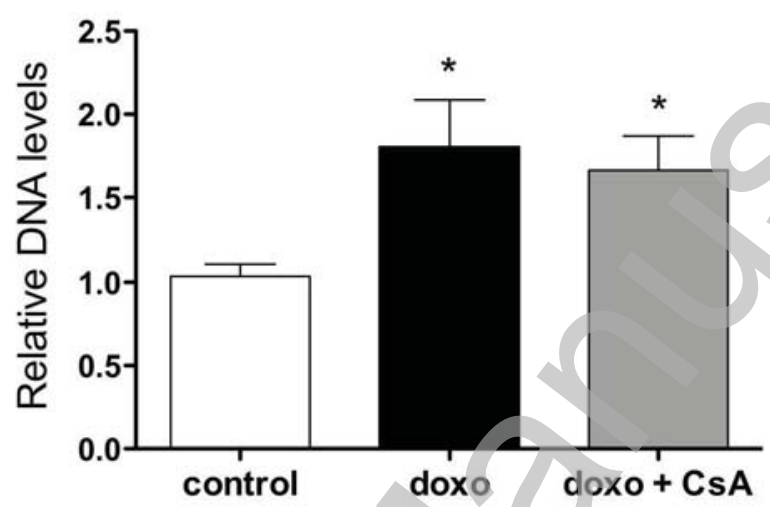

$\mathbf{B}$

PGC1 $\alpha$

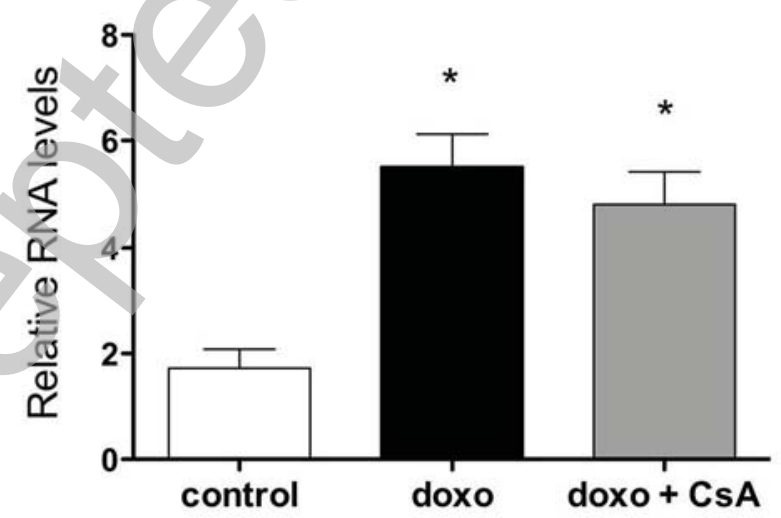


A

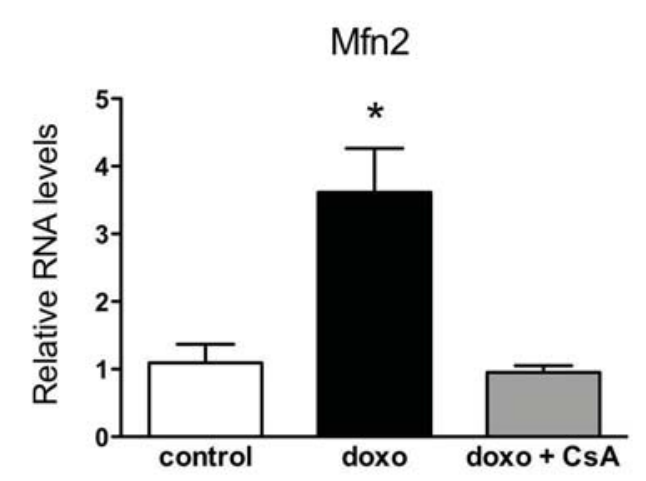

C

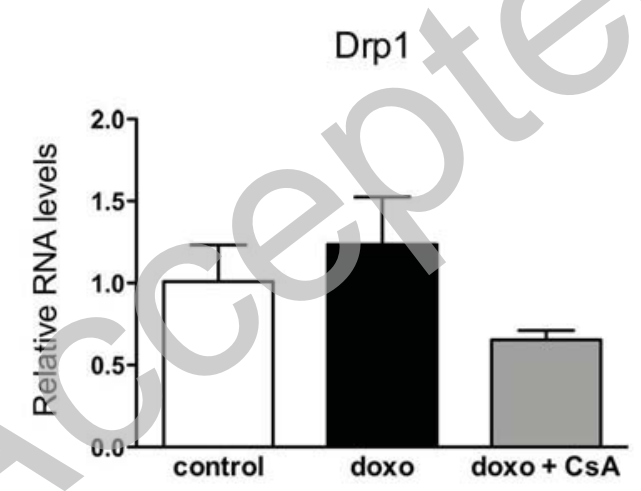

B

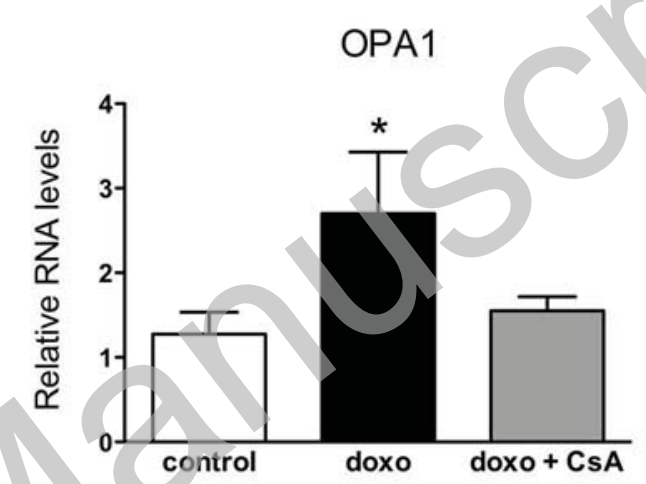

D

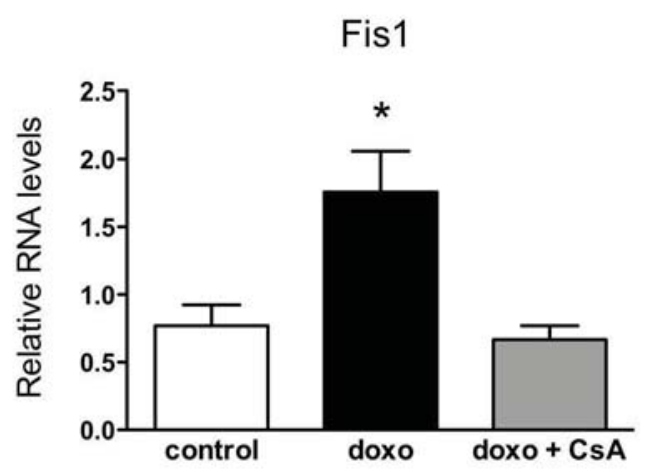

